\title{
Editorial on Advances in Machine Learning and Robotics
}

\section{Manu Mitra}

Department of Electrical Engineering, Alumnus of University of Bridgeport, Bridgeport, United States. manu.ieee@gmail.com

\section{INTRODUCTION}

Machine Learning is opening door to entirely new automation possibilities. It is set to disrupt practically every industry imaginable. Presently, machine learning are being applied in limited methods and are enhancing the abilitiesof industrial robotic systems. There is always room for improvement for the full potential of robotics and machine learning but applications are advantageous. There are four major areas for robotic process and machine learning are impacting to make current applications more efficient and beneficial. It includes Vision - machine learning is aiding robots to detect items that they have never seen before and analyze objects with greater detail. Grasping - robots are also gripping objects that they have never seen before with machine learning it is assisting them to determine the best position and orientation to hold the object. Motion Control with the assistance of machine learning robots are able to have dynamic interaction and obstacle avoidance to maintain productivity. Data - with the help of machine learning and artificial intelligence robot can understand physical and logistical data patterns to be proactive and act appropriately. [1]

\section{MaCHine LEARNing FOR ACCURATE EXTRAPolation}

In past, machine learning was only able to interpolating data; making estimations about situations that are "between" and other known conditions. It was not able to extrapolating - making calculations about situations outside of the known; the reason because it learns to fit the known data as narrowly as possible locally, regardless of how it performs outside of these conditions. In addition to that collecting sufficient data for effective interpolation is time and resource intensive and requires data from very dangerous or extreme conditions. But now, scientist at Institute of Science and Technology Austria has created a machine learning method to accurately extrapolate to unseen conditions. The vital piece of this novel method is that it strives to disclose the true dynamics of the condition: it takes in data and returns the equations that defines the underlying physics. To paraphrase it, this method allows extrapolate reliably, making it unique among machine learning methods. Final approximations that previously generated during machine learning were too complex for human to understand or work with it. But in this new method, the result equations were far simpler to understand. This new method is simpler; which means that less data is required to give same or even better results. Scientists are also exploring the concept whether robots can model not just their own bodies, but also their own minds, whether robots can think about thinking. [2, 3]

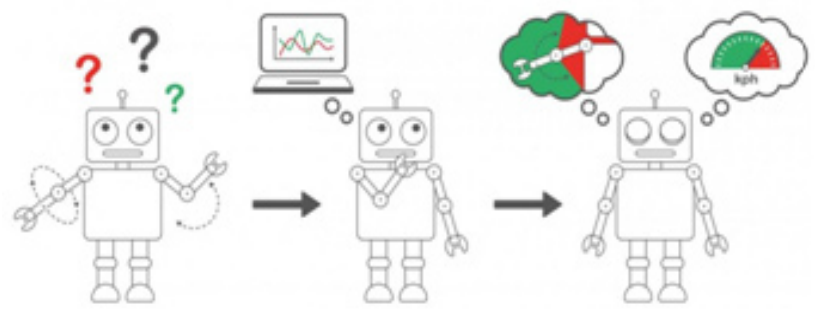

Fig1. Illustrates a robot needs to learn about his body and the environment. It tries a few different motions and uses the algorithm. It can then predict what will happen with larger movements and at higher speeds. Image Credit: IST Austria/Birgit Rieger [3] 


\section{ROBOTS THAT CAN IMAGINE ITSELF}

Researchers at Columbia University School of Engineering and Applied Science made a major advance in robotics by creating a robot that learns what it is, from scratch with zero previous knowledge of geometry, physics or motor dynamics. For instance robot does not know if it's a snake, spider, it has no clue what size or shape it is. After period of time and within about a day of intensive computing, the robot creates self-simulation. This robot uses self-simulator internally to anticipate and familiarize to different environment conditions, handling new series of errands as well as detecting and repairing damage in its own body. Even in the open-loop system that involves series of errands based completely on the internal self-model, without any external feedback, the robot was able to complete pick and place work with a success rate of 44 percent. In this experiments performed selfmodeling robot was also used for other responsibilities such as writing text using a marker. To check whether the self-model could detect damage to itself; scientists 3D printed a deformed part to imitate damage and robot was able to detect the change and re-train its self-model. The new self-model enabled the robot to resume its pick and place series of errands with little loss of performance. $[4,5]$

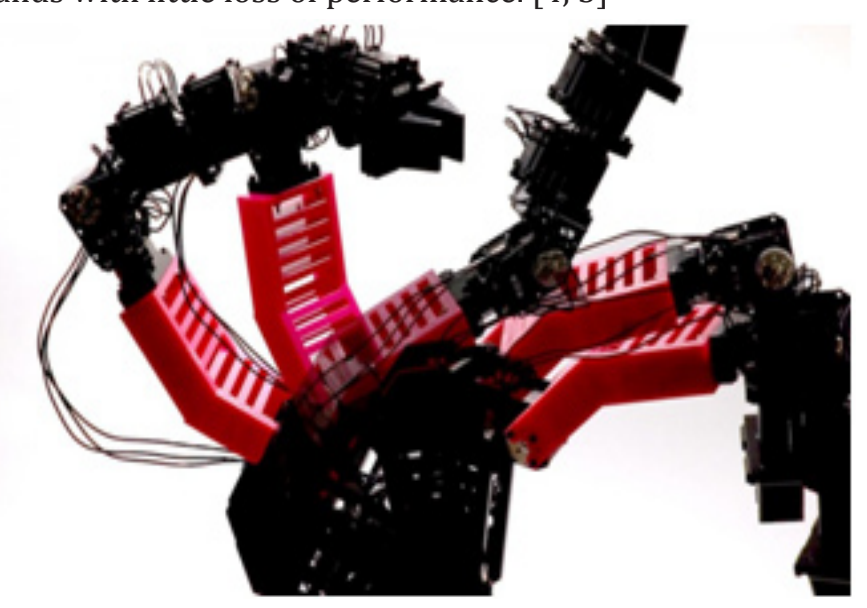

Fig2. Illustrates image of the deformed robotic arm in multiple poses as it was collecting data through random motion. Image Credit: Robert Kwiatkowski/Columbia Engineering [5]

\section{Deep Learning and Big Data to Create Music}

Scientists were able to set 5000 complete songs in a robot - from Beethoven to Beatles to Lady Gaga to Miles Davis and more. Besides, giving machine more data; the first four measures used as starting point and no humans were involved in either the composition or performance of the music. This is the first time a robot is used for deep learning to create music and robot played monophonically, Shimon was able to play harmonies and chords. It's also thinking like a human musician, focusing less on the next note as it did before on the overall structure of the composition. Shimon was created by Bretan's advisor, Gil Weinberg, director of Georgia Tech's Center for Music Technology.Shimon will be able to create more various pieces in the future. As long as experts set it a different input, the robot will generate something different each time - music that scientists can't predict. In the first piece, Bretan gave the input to Shimon a melody comprised of eight notes. It acknowledged sixteenth note melody which influenced it to generate faster note sequences. Shimon's debut as a solo composer was presented in Consumer Electronic Show (CES) keynote and had its live performance at Aspen Ideas Festival. Researchers also created a robotic prosthesis for a drummer - a robotic third arm for all drummers and a collaborative robotic companion that plays music from a phone and dances to the beat. [6] 


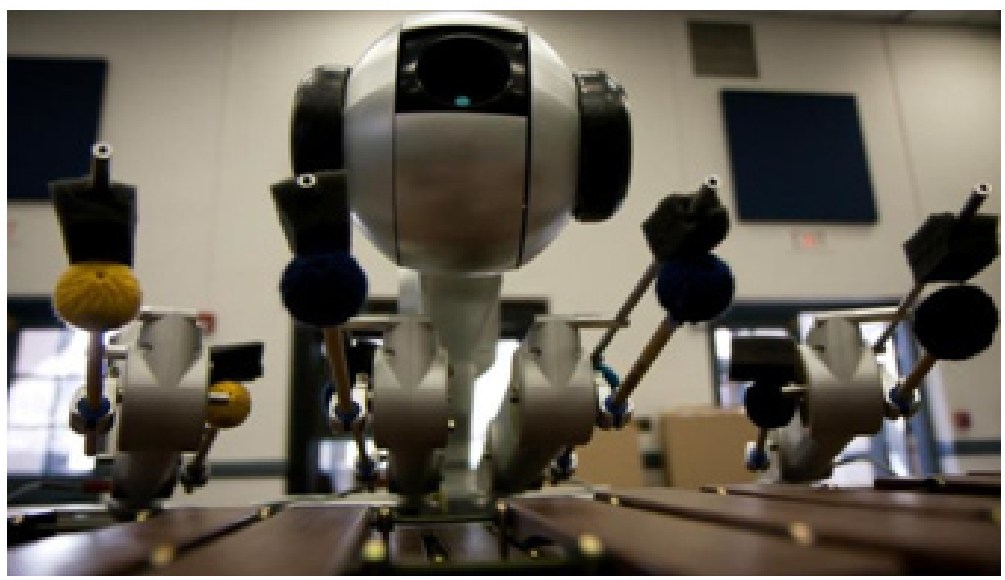

Fig3. Illustrates Shimon, a robot in the Center of Music Technology and School of Music. Image Credit: Georgia Institute of Technology [6]

\section{A Molecular Robot that Can Build Molecules}

Researchers at University of Manchester created world's first "molecular robot" that can perform basic job including building other molecules. These minute robots which are millionth of a millimeter in size can be programmed to move and create molecular cargo using a tiny robotic arm. Each individual robot can manipulate a single molecule and is made up of just 150 carbon, oxygen, hydrogen and nitrogen atoms. To paraphrase it, a billion of billion robot piled on top of each other would still only be the same size as a single grain of salt. These robots function out on chemical reactions in special solutions which can be controlled and programmed by experts to perform basic and series of errands. The advantage of having such kind of technology that is so small is: it massively reduces demand for materials and speedup and advance in drug discovery, drastically reducing power requirements and briskly increase the miniaturization of other products. The potential applications for molecular robots are tremendously varied and exciting. Nevertheless, building and operating such tiny machines are extremely complex, techniques used by the team are based on simple chemical process. $[7,8]$

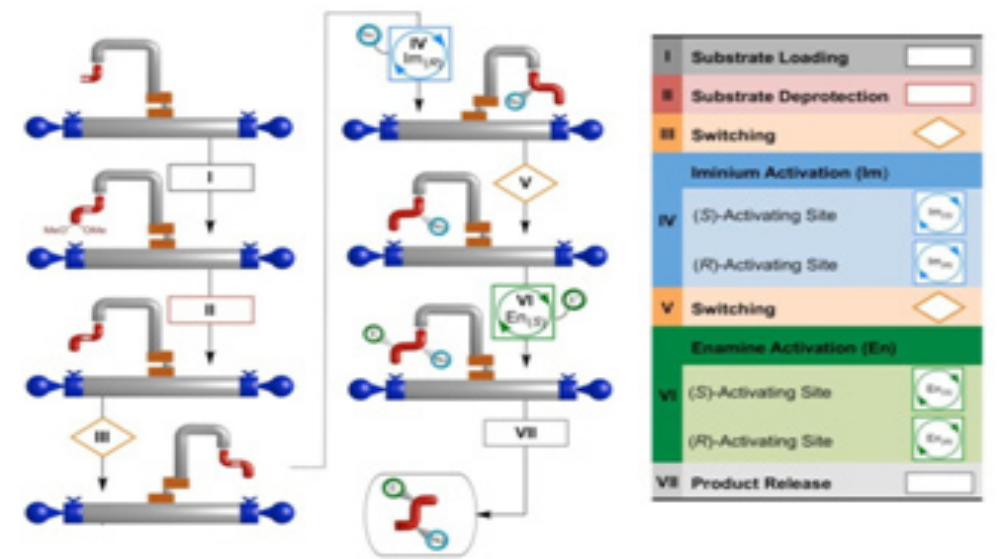

Fig4. Illustrates Molecular robotics represents the ultimate in the miniaturization of machinery, researchers say. Image Credit: University of Manchester [8]

\section{Machine Learning in Plastic Surgery}

With ever growingcapacity of automatic data collected by the health care system, scientist are making use of machine learning to improve medical care and patient outcomes. New advancements of novel method could contribute to advancements in plastic surgery. Machine learning analyzes historical data to create algorithms 
that are capable enough for knowledge acquisition. Authors highlight five major areas where machine learning demonstrates efficiency and clinical outcomes: Brain Surgery - machine learning approach have been created to calculate the healing time of burns providing an effective tool for evaluating burn depth. Algorithms can be created to enable rapid calculations of percentage of body surface burned; a very critical piece of information for patient recovery and surgical planning. Microsurgery -a postoperative microsurgery application has been created to monitor blood perfusion of tissue, flaps based on photographs. In future, algorithms can be created to aid in reconstructive surgery method for individual patients. Craniofacial Surgery - machine learning method for automated diagnosis of infant skull growth defects has been created. Future algorithms can be useful for identifying known and unknown genes accountable for cleft lip and palate. Peripheral Nerve and Hand Surgery machine learning method are useful in calculating success of tissue engineered nerve grafts, creating automated controllers for hand and arm neuro prostheses in patients with spinal cord injuries and improved planning and outcome calculation in hand surgery. Aesthetic Surgery - machine learning also has potential applications in cosmetic surgery, for instance calculating and imitating the outcomes of aesthetic facial surgery. $[9,10]$

\section{CONCLUSION}

To conclude this editorial paper discusses on various applications of robotics and machine learning. Although, this paper being very short survey of robotics and machine learning; it gives brief insight of advances and developments of machine learning. More advantages, applications, methods and novelty can be developed and formulated and moving forward with the development and improving not only in economic conditions but also in standard of livingand conveniences.

\section{ACKNOWLEDGMENT}

Author would like to thank Prof. Navarun Gupta, Prof. Hassan Bajwa, Prof. Linfeng Zhang and Prof. Hmurcik for their academic support. Author also thanks anonymous reviewers for their comments.

\section{REFERENCES}

1. Robotics Online. (2018, June 26). Industrial Robotics and Automation Blog. Retrieved from https://www. robotics.org/blog-article.cfm/Applying-Artificial-Intelligence-and-Machine-Learning-in-Robotics/

2. Subham S. Sahoo, Christoph H. Lampert, Georg Martius. Learning Equations for Extrapolation and Control. 35th International Conference on Machine Learning, 2018

3. Institute of Science and Technology Austria. (2018, July 12). First machine learning method capable of accurate extrapolation. ScienceDaily. Retrieved March 7, 2019 from www.sciencedaily.com/releases/ 2018/07/180712123938.htm

4. Robert Kwiatkowski, Hod Lipson. Task-agnostic self-modeling machines. Science Robotics, 2019; 4 (26): eaau9354 DOI: 10.1126/scirobotics.aau9354

5. Columbia University School of Engineering and Applied Science. (2019, January 30). Engineers create a robot that can 'imagine' itself. ScienceDaily. Retrieved March 7, 2019 from www.sciencedaily.com/ releases/2019/01/190130175621.htm

6. Georgia Institute of Technology. (2017, June 14). Robot uses deep learning and big data to write and play its own music. ScienceDaily. Retrieved March 7, 2019 from www.sciencedaily.com/ releases/2017/06/170614120407.htm

7. Salma Kassem, Alan T. L. Lee, David A. Leigh, Vanesa Marcos, Leoni I. Palmer, Simone Pisano. Stereodivergent synthesis with a programmable molecular machine. Nature, 2017; 549 (7672): 374 DOI: 10.1038/ nature23677

American Research Journal of Electronics and Communication Engineering

Page 19 
Editorial on Advances in Machine Learning and Robotics

8. University of Manchester. (2017, September 20). World's first'molecular robot' capable of building molecules. ScienceDaily. Retrieved March 7, 2019 from www.sciencedaily.com/releases/2017/09/170920131744. htm

9. Jonathan Kanevsky, Jason Corban, Richard Gaster, Ari Kanevsky, Samuel Lin, MirkoGilardino. Big Data and Machine Learning in Plastic Surgery. Plastic and Reconstructive Surgery, 2016; 137 (5): 890e DOI: 10.1097/ PRS.0000000000002088

10. Wolters Kluwer Health. (2016, April 29). 'Machinelearning' may contribute to new advances in plastic surgery. ScienceDaily. Retrieved March 6, 2019 from www.sciencedaily.com/releases/2016/04/160429192812. htm

Citation: Manu Mitra, "Editorial on Advances in Machine Learning and Robotics". American Research Journal of Electronics and Communication Engineering; vol 1, no. 1; pp: 16-20.

Copyright (C) Manu Mitra. This is an open access article distributed under the Creative Commons Attribution License, which permits unrestricted use, distribution, and reproduction in any medium, provided the original work is properly cited. 